PROCEEDINGS OF THE

AMERICAN MATHEMATICAL SOCIETY

Volume 130, Number 10, Pages 2959-2970

S 0002-9939(02)06433-X

Article electronically published on March 12, 2002

\title{
ADMISSIBLE VECTORS FOR THE REGULAR REPRESENTATION
}

\author{
HARTMUT FÜHR
}

(Communicated by David R. Larson)

\begin{abstract}
It is well known that for irreducible, square-integrable representations of a locally compact group, there exist so-called admissible vectors which allow the construction of generalized continuous wavelet transforms. In this paper we discuss when the irreducibility requirement can be dropped, using a connection between generalized wavelet transforms and Plancherel theory. For unimodular groups with type I regular representation, the existence of admissible vectors is equivalent to a finite measure condition. The main result of this paper states that this restriction disappears in the nonunimodular case: Given a nondiscrete, second countable group $G$ with type I regular representation $\lambda_{G}$, we show that $\lambda_{G}$ itself (and hence every subrepresentation thereof) has an admissible vector in the sense of wavelet theory iff $G$ is nonunimodular.
\end{abstract}

\section{INTRODUCTION}

This paper deals with the group-theoretic approach to the construction of continuous wavelet transforms. Shortly after the continuous wavelet transform of univariate functions had been introduced, Grossmann, Morlet and Paul established the connection to the representation theory of locally compact groups [12, which was then used by several authors to construct higher-dimensional analogues 19, 5, 4, 10, 14.

Let us roughly sketch the general group-theoretic formalism for the construction of wavelet transforms. Given a unitary representation $\pi$ of the locally compact group $G$ on the Hilbert space $\mathcal{H}_{\pi}$, and given a vector $\eta \in \mathcal{H}_{\pi}$ (the representation space of $\pi$ ), we can study the mapping $V_{\eta}$, which maps each $\phi \in \mathcal{H}_{\pi}$ to the bounded continuous function $V_{\eta} \phi$ on $G$, defined by

$$
V_{\eta} \phi(x):=\langle\phi, \pi(x) \eta\rangle \text {. }
$$

Whenever this operator $V_{\eta}$ is an isometry of $\mathcal{H}_{\pi}$ into $\mathrm{L}^{2}(G)$, we call it a continuous wavelet transform, and $\eta$ is called a wavelet or admissible vector. The construction has also been studied in mathematical physics, where admissible vectors are known under the name coherent states.

Note that by its very definition a wavelet transform is an intertwining operator between $\pi$ and $\lambda_{G}$, the left regular representation. The observation made in

Received by the editors October 26, 2000 and, in revised form, May 3, 2001.

2000 Mathematics Subject Classification. Primary 43A30; Secondary 42C40.

Key words and phrases. Continuous wavelet transforms, coherent states, square-integrable representations, Plancherel theory, cyclic vectors. 
12 was that admissible vectors always exist, when $\pi$ is (unitarily equivalent to) an irreducible subrepresentation of the regular representation of $G$. Such representations are usually called "square-integrable" or "discrete series representations". This covers both the standard continuous wavelet transform on $\mathrm{L}^{2}(\mathbb{R})$, where the underlying group is the affine group of the real line, and the windowed Fourier (or Gabor) transform, based on an irreducible representation of the Weyl-Heisenberg group.

While this formalism is flexible enough to allow for a variety of transforms (as documented by the above-cited higher-dimensional analogues), several approaches exist to construct transforms in more general settings: On the one hand, the squareintegrability requirement can be replaced by square-integrability on quotients (see 1] for an exposition of these techniques).

On the other hand, certain nonirreducible representations have been considered as well [13, 15], indicating that the irreducibility requirement can be weakened. Another step in this direction is taken in [16, which studies in full generality the quasiregular representation of a semidirect product $\mathbb{R}^{n} \rtimes H$ on $\mathrm{L}^{2}\left(\mathbb{R}^{n}\right)$, where $H$ is a closed matrix group, with the aim of establishing admissibility conditions. [16] gives an almost complete characterization of the matrix groups $H$ for which an admissible vector exists; only for a small part of those groups the quasiregular representation is in fact irreducible.

These examples indicate a growing interest in wavelet transforms arising from reducible representations, and they serve as motivation for this paper. We give a complete characterization of the subrepresentations of the left regular representation of a locally compact group allowing admissible vectors, whenever the regular representation is type I. The criteria are given in terms of the Plancherel measure of the group. Since we are dealing with subrepresentations of the regular representation, it seems quite natural to employ the decomposition of the regular representation into irreducibles, that is, the Plancherel decomposition. The connection was already noted by Carey [6], but had not been further pursued. To motivate the approach via Plancherel theory, let us consider the following toy example:

Example 0.1. Let $G=\mathbb{R}$, and let $\mathcal{H} \subset \mathrm{L}^{2}(\mathbb{R})$ be a translation-invariant closed subspace. Then there exists a measurable subset $U \subset \mathbb{R}$ such that

$$
\mathcal{H}=\left\{f \in \mathrm{L}^{2}(\mathbb{R}): \widehat{f} \text { vanishes outside } U\right\} .
$$

The admissible vectors are easily identified with the aid of the Fourier transform: For $\eta, \phi \in \mathcal{H}$ we have $V_{\eta} \phi=\phi * \tilde{\eta}$, with $\tilde{\eta}(x)=\overline{\eta(-x)}$, and hence $\widehat{V_{\eta} \phi}(\omega)=$ $\widehat{\phi}(\omega) \bar{\eta}(\omega)$. Thus, $\eta$ is admissible for $\mathcal{H}$ iff $|\widehat{\eta}(\omega)|$ equals one almost everywhere on $U$, and such vectors exist iff $U$ has finite Lebesgue measure.

Another observation will be useful for the following: Denote the restriction of the regular representation to $\mathcal{H}$ by $\pi$ and let $\widehat{\pi}$ denote the representation obtained by conjugating $\pi$ with the Fourier transform, so that $\widehat{\pi}$ operates on $\mathrm{L}^{2}(U)$ by $\widehat{\pi}(x) f(\omega)=e^{i \omega x} f(\omega)$. If we choose two vectors $\eta, \phi \in \mathrm{L}^{2}(U)$, then we see that

$$
\left(V_{\eta} \phi\right)(x)=\int_{U} \phi(\omega) \overline{\eta(\omega)} e^{-i \omega x} d \omega,
$$

i.e., in this realization of the representation, the wavelet transform is just a special instance of Fourier inversion. 
It turns out that this argument generalizes almost verbatim to the case of unimodular locally compact groups with type I regular representation. Just as for $G=\mathbb{R}$, the Plancherel decomposition of such a group allows:

- direct access to all invariant subspaces of $\mathrm{L}^{2}(G)$;

- to convert convolution operators to pointwise multiplication operators; and thus:

- to construct admissible vectors on the Plancherel transform side; and finally:

- to classify the subspaces having admissible vectors by a finite measure condition.

The classification of the subspaces with admissible vectors can be found in Theorem 1.6 below. The only price we have to pay for passing from abelian to nonabelian unimodular groups is that irreducible representations are no longer one-dimensional. Hence we have to deal with multiplicities in the Plancherel decomposition, and to replace scalar multiplication on the Fourier side by operator multiplication.

However, when we consider nonunimodular groups, the situation changes drastically. The complications arise from the family of unbounded operators intervening in the Plancherel transform, the so-called Duflo-Moore or formal degree operators. These operators also show up in the decomposition of convolution operators. However, it turns out that their unboundedness can be exploited to construct admissible vectors for arbitrary subrepresentations of the regular representation. This includes the regular representation itself, and in fact it is enough to concentrate on this case. These will be the main results of this paper:

Theorem 0.2. Let $G$ be nonunimodular with type I regular representation $\lambda_{G}$. Then $\lambda_{G}$ has an admissible vector.

Corollary 0.3. Let $G$ be nonunimodular with type I regular representation. A representation $\pi$ of $G$ has admissible vectors iff $\pi$ is equivalent to a subrepresentation of $\lambda_{G}$.

Proof. Only the "if" part remains to be shown. So let $\mathcal{H}_{\pi} \subset \mathrm{L}^{2}(G)$ be a leftinvariant subspace. Let $g$ be an admissible vector for the regular representation, provided by Theorem 0.2 If $P$ denotes the projection into $\mathcal{H}_{\pi}$, then $P g$ is an admissible vector for $\mathcal{H}_{\pi}$, since $V_{g} f=V_{P g} f$, for all $f \in \mathcal{H}_{\pi}$.

Theorem 0.2 is all the more surprising, as the contrary is proved for unimodular groups - excepting the (trivial) case of discrete groups - with great ease, and without the use of Plancherel theory:

Proposition 0.4. Let $G$ be a unimodular group, such that $\lambda_{G}$ has an admissible vector. Then $G$ is discrete.

Proof. Suppose $g$ is an admissible vector. Then $V_{g} f=f * \tilde{g}$, and the adjoint of $V_{g}$ is given by $V_{\tilde{g}}$, where $\tilde{g}(x)=\overline{g\left(x^{-1}\right)}$, which defines an $\mathrm{L}^{2}$ function (here we use unimodularity). Hence, for every $f \in \mathrm{L}^{2}(G), f=V_{\tilde{g}}\left(V_{g} f\right)$, and the right-hand side is a continuous function. Hence for every $\mathrm{L}^{2}$-function $f$ there exists a continuous function which coincides with $f$ almost everywhere. But then $G$ is discrete.

For unimodular groups with noncompact connected component, we can sharpen the proposition some more: From [3, Theorem 1.3] it follows that if $\mathcal{H} \subset \mathrm{L}^{2}(G)$ has an admissible vector, then $\mathcal{H}$ contains no nontrivial elements supported in a set of finite measure. 
The proof of the proposition in fact shows that, for any admissible vector $g$ for the regular representation of an arbitrary locally compact group $G, \tilde{g}$ cannot be in $\mathrm{L}^{2}(G)$. This indicates that the direct construction of admissible vectors will be difficult; for one thing, admissible vectors are not compactly supported.

Let us fix some notation: $G$ denotes a locally compact, second countable group. Its left Haar measure is denoted by $\mu_{G}, \mathrm{~L}^{2}(G)$ is the corresponding $\mathrm{L}^{2}$-space. $\lambda_{G}$ and $\rho_{G}$ are the left respectively right regular representation; we always assume that $\lambda_{G}$ is type I. $\Delta_{G}$ denotes the modular function of $G$. Representations are always understood to be strongly continuous and unitary. $\widehat{G}$ denotes the space of equivalence classes of irreducible representations of $G$ endowed with the Mackey Borel structure. We will not explicitly distinguish between representations and equivalence classes.

For a separable Hilbert space $\mathcal{H}$, we let $\operatorname{dim}(\mathcal{H})$ denote its Hilbert space dimension. $\mathcal{B}_{2}(\mathcal{H})$ denotes the space of Hilbert-Schmidt operators on $\mathcal{H}$. The norm on $\mathcal{B}_{2}(\mathcal{H})$ is denoted by $\|\cdot\|_{2}$. The usual operator norm is denoted by $\|\cdot\|_{\infty}$. If an operator $A$ is densely defined on $\mathcal{H}$ and has a bounded extension to all of $\mathcal{H}$, we denote this extension by $[A]$, and we say that " $[A]$ exists".

Before we enter the discussion of Plancherel theory, a comment on the type I condition on $\lambda_{G}$ is in order. Technically speaking, it is a condition on the von Neumann algebra generated by $\lambda_{G}(G)$ []. Instead of reproducing that definition, let us just say that it is fulfilled for large classes of groups, such as semisimple or nilpotent connected Lie groups, or real algebraic groups. In fact for these groups every representation is type I; confer [9, Theorem 7.8]. Also, $\lambda_{G}$ is (quite trivially) type I whenever it is the direct sum of irreducibles, as is the case for the $a x+b$-group.

\section{Plancherel theory}

In this section we give a short account of Plancherel theory, which we then use to reduce the problem of finding admissible vectors to the construction of certain operator fields. The starting point for the definition of the Plancherel transform is the operator valued Fourier transform on $\mathrm{L}^{1}(G)$. Given $f \in \mathrm{L}^{1}(G)$ and $\sigma \in \widehat{G}$, we define

$$
\mathcal{F}(f)(\sigma):=\sigma(f):=\int_{G} f(x) \sigma(x) d \mu_{G}(x),
$$

where the integral is taken in the weak operator sense. As direct consequences of the definition we have $\|\sigma(f)\|_{\infty} \leq\|f\|_{1}$ and $\sigma(f * g)=\sigma(f) \circ \sigma(g)$.

The Plancherel transform is obtained by extending the Fourier transform from $\mathrm{L}^{1}(G) \cap \mathrm{L}^{2}(G)$ to $\mathrm{L}^{2}(G)$. The nonunimodular part of the following Plancherel theorem is due to Duflo and Moore [8, Theorem 5], whereas the unimodular version may be found in 7 .

Theorem 1.1. Let $G$ be a second countable locally compact group having a type I regular representation. Then there exists a measure $\nu_{G}$ on $\hat{G}$ and a measurable field $\left(C_{\sigma}\right)_{\sigma \in \hat{G}}$ of selfadjoint positive operators with densely defined inverse, with the following properties:

(i) For $f \in \mathrm{L}^{1}(G) \cap \mathrm{L}^{2}(G)$ and $\nu_{G}$-almost all $\sigma \in \hat{G}$, the closure of the operator $\sigma(f) C_{\sigma}^{-1}$ is a Hilbert-Schmidt operator on $\mathcal{H}_{\pi}$. 
(ii) The map $\mathrm{L}^{1}(G) \cap \mathrm{L}^{2}(G) \ni f \mapsto\left(\left[\sigma(f) C_{\sigma}^{-1}\right]\right)_{\sigma \in \widehat{G}}$ extends to a unitary equivalence

$$
\mathcal{P}: \mathrm{L}^{2}(G) \rightarrow \mathcal{B}_{2}^{\oplus}:=\int_{\hat{G}}^{\oplus} \mathcal{B}_{2}\left(\mathcal{H}_{\sigma}\right) d \nu_{G}(\sigma) .
$$

This unitary operator is called the Plancherel transform of $G$. It intertwines the two-sided representation $\lambda_{G} \times \rho_{G}$ with $\int_{\hat{G}}^{\oplus} \sigma \otimes \bar{\sigma} d \nu_{G}(\sigma)$.

(iii) $G$ is unimodular iff $\nu_{G}$-almost all $C_{\sigma}$ are scalar multiples of the identity operator. In this case we fix $C_{\sigma}=\mathrm{Id}_{\mathcal{H}_{\sigma}}$, and then the measure $\nu_{G}$ is uniquely determined.

In the following $\widehat{f}$ denotes the Plancherel transform of the $\mathrm{L}^{2}$-function $f$; in particular in the nonunimodular case it should not be confused with the Fourier transform. Note that our terminology differs in two ways from [8]: We denote the closure of an operator $A$ by $[A]$, and we use slightly different operators. Our $C_{\sigma}$ and the operators $K_{\sigma}$ in [8] are related by $C_{\sigma}=K_{\sigma}^{-1 / 2}$.

In the nonunimodular case, almost every $C_{\sigma}$ is an unbounded operator (this will become clear in Section 2 below), and it is only fixed up to a constant multiple. Since there is apparently no natural choice of normalization, there is also no canonical choice of the measure $\nu_{G}$, which is only unique up to equivalence.

A further important feature of the Plancherel transform is the decomposition of intertwining operators: If $T: \mathrm{L}^{2}(G) \rightarrow \mathrm{L}^{2}(G)$ is a bounded operator which commutes with left translations, then there exists a measurable field of bounded operators $\left(T_{\sigma}\right)_{\sigma \in \widehat{G}}$ with $\left\|T_{\sigma}\right\|_{\infty}$ uniformly bounded, such that

$$
T=\int_{\widehat{G}}^{\oplus} \operatorname{Id}_{\mathcal{H}_{\sigma}} \otimes T_{\sigma} d \nu_{G}(\sigma) .
$$

This applies in particular to the projection onto invariant subspaces. The obvious analogue for the right action of $G$ holds as well.

As explained earlier we are interested in constructing admissible vectors on the Plancherel transform side. Hence it is necessary to have a pointwise Fourier inversion formula. The following theorem is proven in 2. It can be seen as a generalization of [17] Theorem 4.1] or as the nonabelian analogue of the following simple fact from abelian Fourier analysis: Given an $\mathrm{L}^{2}$-function $f$ whose Plancherel transform $\hat{f}$ is in $\mathrm{L}^{1}$, then, pointwise almost everywhere, $f$ equals the Fourier transform of $\widetilde{\hat{f}}$.

Theorem 1.2. Let $A \in \mathcal{B}_{2}^{\oplus}$ be such that for almost all $\sigma \in \hat{G}, A(\sigma) C_{\sigma}^{-1}$ extends to a trace-class operator. Suppose moreover that the mapping $\sigma \mapsto \operatorname{tr}\left(\left|\left[A(\sigma) C_{\sigma}^{-1}\right]\right|\right)$ is in $\mathrm{L}^{1}\left(\hat{G}, d \nu_{G}\right)$. Let $a \in \mathrm{L}^{2}(G)$ be the inverse Plancherel transform of $A$. Then we have (almost everywhere)

$$
a(x)=\int_{\hat{G}} \operatorname{tr}\left(\left[A(\sigma) C_{\sigma}^{-1}\right] \sigma(x)^{*}\right) d \nu_{G}(\sigma) .
$$

Remark 1.3. Let us now briefly explain how wavelet transforms associated to irreducible representations relate to Plancherel transform. So let $\pi<\lambda_{G}$ be an irreducible subrepresentation; we may assume that $\pi$ is also used in the Plancherel deomposition. Then $\nu_{G}(\{\pi\})>0$, and by suitably normalizing the operator $C_{\pi}$ we can assume that $\nu_{G}(\{\pi\})=1$. As is well-known, the admissible vectors are those in $\operatorname{dom}\left(C_{\pi}\right)$. So let $\eta \in \operatorname{dom}\left(C_{\pi}\right)$, and associate to $\phi \in \mathcal{H}_{\pi}$ the rank-one operator $A_{\pi}=\phi \otimes C_{\pi} \eta$. If we denote by $A \in \mathcal{B}_{2}^{\oplus}$ the operator field which is $A_{\pi}$ at $\pi$ and 
zero elsewhere, we find that Theorem 1.2 is applicable and gives for the inverse Plancherel transform $a$ of $A$

$$
\begin{aligned}
a(x) & =\int_{\hat{G}} \operatorname{tr}\left(\left[A(\sigma) C_{\sigma}^{-1}\right] \sigma(x)^{*}\right) d \nu_{G}(\sigma) \\
& =\operatorname{tr}\left(\left(\phi \otimes C_{\pi} \eta\right) C_{\pi}^{-1} \pi(x)^{*}\right) \\
& =\operatorname{tr}(\phi \otimes \pi(x) \eta) \\
& =\langle\phi, \pi(x) \eta\rangle \\
& =V_{\eta} \phi(x) .
\end{aligned}
$$

Hence $A=\left(V_{\eta} \phi\right)^{\wedge}$, and the isometry properties of $V_{\eta}$ are easily recognised as special instances of the unitarity of Plancherel transform.

For multiplicity-free representations, this way of arguing can be immediately generalized, and one obtains admissibility conditions which are fairly easy to handle. The multiplicity-free case covers for instance the quasi-regular representations of semidirect products of $\mathbb{R}^{k}$ with a closed matrix group, acting on $\mathrm{L}^{2}\left(\mathbb{R}^{k}\right)$. In particular the results obtained in 13, 15 can be interpreted under this perspective.

But also for representations with multiplicities, in particular the regular representation itself, one can derive sufficient admissibility conditions, which reduce the construction of admissible vectors to the construction of certain operator fields. This is the subject of the next lemma.

Lemma 1.4. Suppose that $g \in \mathrm{L}^{2}(G)$ is such that $\left[\hat{g}(\sigma)^{*} C_{\sigma}\right]$ exists, for almost every $\sigma \in \hat{G}$, and that the mapping $\sigma \mapsto\left\|\left[\hat{g}(\sigma)^{*} C_{\sigma}\right]\right\|_{\infty}$ is bounded $\nu_{G}$-almost everywhere. Then, for all $f \in \mathrm{L}^{2}(G), V_{g} f \in \mathrm{L}^{2}(G)$, and

$$
\left(V_{g} f\right)^{\wedge}(\sigma)=\hat{f}(\sigma)\left[\hat{g}(\sigma)^{*} C_{\sigma}\right] .
$$

Proof. For $f \in \mathrm{L}^{2}(G)$, consider the measurable field of operators $(A(\sigma))_{\sigma \in \hat{G}}$, defined by $A(\sigma)=\hat{f}(\sigma)\left[\hat{g}(\sigma)^{*} C_{\sigma}\right]$. Then, by the boundedness condition, $A \in \mathcal{B}_{2}^{\oplus}$. Moreover $\left[A(\sigma) C_{\sigma}^{-1}\right]=\hat{f}(\sigma) \hat{g}(\sigma)^{*}$ exists and defines a field of trace class operators with integrable trace class norm. Hence we may apply the inversion formula and obtain for the inverse Plancherel transform $a$ of $A$ :

$$
a(x)=\int_{\hat{G}} \operatorname{tr}\left(\hat{f}(\sigma) \hat{g}(\sigma)^{*} \sigma(x)^{*}\right) d \nu_{G}(\sigma)=\left\langle f, \lambda_{G}(x) g\right\rangle .
$$

Lemma 1.4 gives a sufficient criterion for admissibility: We have to construct $(A(\sigma))_{\sigma \in \hat{G}} \in \mathcal{B}_{2}^{\oplus}$ in such a way that $B \mapsto B\left[A(\sigma)^{*} C_{\sigma}\right]$ defines an isometry in $\mathcal{B}_{2}\left(\mathcal{H}_{\sigma}\right)$, for almost every $\sigma \in \hat{G}$. The latter is easily seen to be equivalent to the fact that $\left[A(\sigma)^{*} C_{\sigma}\right]^{*}$ is an isometry. The inverse Plancherel transform of such an operator field is then the desired admissible vector. At this stage, the effect of nonunimodularity becomes visible: In this case there is a chance that such HilbertSchmidt operators $A(\sigma)$ exist, because $C_{\sigma}$ is unbounded. In the unimodular case the $C_{\sigma}$ disappear, and thus $A(\sigma)$ has to be both Hilbert-Schmidt (in particular compact) and an isometry, which only works if $\mathcal{H}_{\sigma}$ is finite-dimensional.

Now let us consider the unimodular case. Before we prove the criterion which generalizes the toy example from above, we need a converse of Lemma 1.4 The statement is very intuitive and most likely well-known, but it seems to us that a rigorous proof is necessarily somewhat technical, due to the fact that the Plancherel 
transform of an arbitrary $\mathrm{L}^{2}$-function is known only almost everywhere, unlike the Fourier transform.

Lemma 1.5. Let $G$ be unimodular. If $g \in \mathrm{L}^{2}(G)$ is such that $V_{g}$ is a bounded operator on $\mathrm{L}^{2}(G)$, then $\|\widehat{g}(\sigma)\|_{\infty}$ is bounded $\nu_{G}$-almost everywhere, and we have for all $f \in \mathrm{L}^{2}(G)$

$$
\left(V_{g} f\right)^{\wedge}(\sigma)=\widehat{f}(\sigma) \widehat{g}(\sigma)^{*}
$$

$\left(\nu_{G}\right.$-almost everywhere $)$.

Proof. The operator $V_{g}$ commutes with left translation and hence has a decomposition

$$
V_{g}=\int_{\widehat{G}}^{\oplus} \operatorname{Id}_{\mathcal{H}_{\sigma}} \otimes A_{\sigma} d \nu_{G}(\sigma)
$$

with a field $\left(A_{\sigma}\right)_{\sigma \in \widehat{G}}$ of essentially uniformly bounded operators. Of course the aim is to show that $A_{\sigma}=\widehat{g}(\sigma)^{*} \nu_{G}$-almost everywhere. For this purpose let $\left(f_{n}\right)_{n \in \mathbb{N}} \subset$ $\mathrm{L}^{1}(G) \cap \mathrm{L}^{2}(G)$ be a sequence with dense span in $\mathrm{L}^{2}(G)$. Then, since the Plancherel transform also intertwines the representations of the convolution algebra $\mathrm{L}^{1}(G)$ arising from the left action of $G$, we have for all $n \in \mathbb{N}$ that

$$
\widehat{f_{n}}(\sigma) A_{\sigma}=\left(V_{g} f_{n}\right)^{\wedge}(\sigma)=\left(f_{n} * \tilde{g}\right)^{\wedge}(\sigma)=\sigma\left(f_{n}\right) \widehat{g}(\sigma)^{*}=\widehat{f_{n}}(\sigma) \widehat{g}(\sigma)^{*},
$$

for all $\sigma$ belonging to a common conull subset $\Sigma \subset \widehat{G}$. Moreover, possibly after passing to a suitable conull subset of $\Sigma$, we can assume that all $A_{\sigma}$ and $\widehat{g}(\sigma)$ are bounded, and that the span of $\left\{\widehat{f_{n}}(\sigma): n \in \mathbb{N}\right\}$ is dense in $\mathcal{B}_{2}\left(\mathcal{H}_{\sigma}\right)$, for every $\sigma \in \Sigma$. But then by the continuity of the operators it follows that $A_{\sigma}=\widehat{g}(\sigma)^{*}$ for all $\sigma \in \Sigma$.

Now we can easily prove the characterization of the subrepresentations of $\lambda_{G}$ with admissible vectors, when $G$ is unimodular. The statement and its proof are somewhat similar to [3, Proposition 1.1]; compare also [6, Theorem 2.10].

Theorem 1.6. Let $G$ be unimodular. Let $\mathcal{H} \subset \mathrm{L}^{2}(G)$ be a left-invariant closed subspace, and let $P$ denote the orthogonal projection onto $\mathcal{H}$. Then

$$
P=\int_{\hat{G}}^{\oplus} \operatorname{Id}_{\mathcal{H}_{\sigma}} \otimes P_{\sigma} d \nu_{G}(\sigma),
$$

with a measurable family of orthogonal projections $\left(P_{\sigma}\right)_{\sigma \in \hat{G}}$. Then $\mathcal{H}$ has admissible vectors iff almost all $P_{\sigma}$ have finite rank and

$$
\nu_{\mathcal{H}}=\int_{\hat{G}} \operatorname{dim}\left(P_{\sigma}\left(\mathcal{H}_{\sigma}\right)\right) d \nu_{G}(\sigma)<\infty .
$$

Every admissible vector $g \in \mathcal{H}$ fulfills $\|g\|^{2}=\nu_{\mathcal{H}}$.

Proof. For the sufficiency we note that $\left(P_{\sigma}\right)_{\sigma \in \hat{G}} \in \mathrm{B}_{2}^{\oplus}$, and we let $g$ be the inverse Plancherel transform of that. Then Lemma 1.4 shows that $P=V_{g}$, which means that $V_{g}$ is the identity operator on $\mathcal{H}$, and $g$ is admissible.

Now let $g$ be an admissible vector for $\mathcal{H}$, and define $h=\tilde{g} * g$. Then we have $P=\left(V_{g}\right)^{*} \circ\left(V_{g}\right)=V_{\tilde{g}} \circ V_{g}=V_{h}$ and $h=V_{\tilde{g}} \tilde{g} \in \mathrm{L}^{2}(G)$ (note that $V_{\tilde{g}}$ is a bounded operator on all of $\mathrm{L}^{2}(G)$ ). Applying Lemma 1.5 first to $V_{h}$ and then to $V_{\tilde{g}}$ yields

$$
P_{\sigma}=\widehat{h}(\sigma)^{*}=\widehat{g}(\sigma)^{*} \widehat{g}(\sigma),
$$


$\nu_{G}$-almost everywhere. Hence

$$
\begin{aligned}
\|g\|^{2} & =\int_{\hat{G}} \operatorname{tr}\left(\hat{g}(\sigma) \hat{g}(\sigma)^{*}\right) d \nu_{G}(\sigma) \\
& =\int_{\hat{G}} \operatorname{tr}\left(\hat{g}(\sigma)^{*} \hat{g}(\sigma)\right) d \nu_{G}(\sigma) \\
& =\int_{\hat{G}} \operatorname{dim}\left(P_{\sigma}\left(\mathcal{H}_{\sigma}\right)\right) d \nu_{G}(\sigma) .
\end{aligned}
$$

The number $\nu_{\mathcal{H}}$ is the "total Plancherel measure" associated to the representation $\left.\lambda_{G}\right|_{\mathcal{H}}$. This terminology expresses the fact that in calculating $\nu_{\mathcal{H}}$, each representation occurring in the direct integral decomposition of $\left.\lambda_{G}\right|_{\mathcal{H}}$ into irreducibles is weighted by its multiplicity, and then integrated against Plancherel measure.

Combining Proposition 0.4 and Theorem 1.6, we find that for nondiscrete unimodular groups the total Plancherel measure of the regular representation itself is always infinite. We expect that this has already been noted elsewhere; it can also be derived from the results in the paper by Arnal and Ludwig [3], where the quantity $\nu_{\mathcal{H}}$ is studied in the context of the so-called qualitative uncertainty property.

\section{Proof of Theorem 0.2}

Throughout this section $G$ is nonunimodular.

We shall now construct the operator field $(\hat{g}(\sigma))_{\sigma \in \hat{G}}$ with the sufficient properties derived from of Lemma 1.4. For this purpose a more detailed knowledge of the operators $C_{\sigma}$ and the representations involved is indispensable, and it can be obtained by considering the closed normal, unimodular subgroup $G_{0}:=\operatorname{Ker}\left(\Delta_{G}\right)$. We denote the quotient $G / G_{0}$ by $\Gamma$ and endow it with the quotient topology. It is thus algebraically (but usually not topologically) isomorphic to some subgroup of $\left(\mathbb{R}^{+}, \cdot\right)$. By abuse of notation, the canonical embedding of $\Gamma$ in $\mathbb{R}^{+}$is also denoted by $\Delta_{G}$.

The main idea pursued in Paragraph 6 of $[8]$ is to perform a Mackey analysis of the group extension $G$ of $G_{0}$. (A similar approach was taken by Tatsuuma [20].) This means that information on the orbit space of the natural action of $G$ (actually, $\Gamma$ ) on the dual $\widehat{G_{0}}$ is utilized to derive the Plancherel theory of $G$ from that of the unimodular subgroup $G_{0}$.

This approach results in a fairly explicit description of the objects involved. More precisely, there exists a $\Gamma$-invariant Borel subset $U \subset \widehat{G_{0}}$, which has the following properties:

(i) It is $\nu_{G_{0}}$-conull in $\widehat{G_{0}}$; here $\nu_{G_{0}}$ is the Plancherel measure of $G_{0}$. The quotient space $U / \Gamma$ is a standard Borel space. (This follows combining [ 8 , Theorem 6 , 1.], [8, Lemma 13] and [8, Corollary 1 to Theorem 6].)

(ii) For every $\sigma \in U, \operatorname{Ind}_{G_{0}}^{G} \sigma \in \widehat{G}[8$, Theorem $6,1$.$] . Hence \Gamma$ operates freely on the orbit $\Gamma \sigma$ [8, Lemma 7].

(iii) Define $V:=\left\{\operatorname{Ind}_{G_{0}}^{G} \rho: \rho \in U\right\}$; then $V$ is a Borel subset of $\hat{G}$ and standard [8, Theorem 6]. By Mackey's theory $V$ is canonically identified with the orbit space $U / \Gamma$, and this identification is a Borel isomorphism [8, Lemma 13]. Moreover there exists a Borel cross section $\tau: V \rightarrow U$, such that $\Gamma \times V \ni$ $(\gamma, \sigma) \mapsto \gamma \tau(\sigma) \in U$ is a Borel isomorphism [8. Proof of Prop. 10]. In 
particular $U$ is standard as well, and hence $\tau(V)$ is standard [18] Theorem $3.2]$.

Using the Borel isomorphism, we transfer the mapping $(\gamma, \sigma) \mapsto \Delta_{G}(\gamma)^{-1 / 2}$ to $U$ and thus obtain a measurable function $\psi$ on $U$. Then $\psi$ obviously fulfills $\psi(\gamma \rho)=\Delta_{G}(\gamma)^{-1 / 2} \psi(\rho)$, for all $\rho \in U$.

(iv) The Plancherel measure is supported by $\operatorname{Ind}(U)$, and can be obtained from the Plancherel measure of $G_{0}$ by measure disintegration.

More precisely: Plancherel measure on $V \cong U / \Gamma$ is obtained from a measure disintegration along $\Gamma$-orbits, as pointed out in $[\underline{8}$, Theorem 6, 3.]. Since $\Gamma$ operates freely, each orbit $\Gamma \tau(\sigma)$ can be endowed with the image $\mu_{\Gamma \tau(\sigma)}$ of Haar measure under the projection map $\gamma \mapsto \gamma \tau(\sigma)$. Then there exists a unique measure $\nu_{G}$ on $V$ such that

$$
d \nu_{G_{0}}(\rho)=\psi(\rho)^{-2} d \mu_{\Gamma \tau(\sigma)}(\rho) d \nu_{G}(\sigma)
$$

the thus found measure $\nu_{G}$ is the Plancherel measure of $G$.

Moreover, the operators $C_{\sigma}$ can be given explicitly in terms of $\Delta_{G}$. Since $\Gamma$ operates freely, we may realize $\sigma=\operatorname{Ind}_{G_{0}}^{G} \tau(\sigma)$ on $\mathrm{L}^{2}\left(\Gamma, d \mu_{\Gamma} ; \mathcal{H}_{\tau(\sigma)}\right)$, and then $C_{\sigma}$ is given by multiplication with $\Delta_{G}^{-1 / 2}$ :

$$
\left(C_{\sigma} \eta\right)(\gamma)=\Delta_{G}(\gamma)^{-1 / 2} \eta(\gamma)
$$

and $\operatorname{dom}\left(C_{\sigma}\right)$ is the set of all $\eta \in \mathrm{L}^{2}\left(\Gamma, d \mu_{\Gamma} ; \mathcal{H}_{\tau(\sigma)}\right)$, for which this product is also in $\mathrm{L}^{2}\left(\Gamma, d \mu_{\Gamma} ; \mathcal{H}_{\tau(\sigma)}\right)$.

At this point it is easy to see that in the nonunimodular case indeed almost every $C_{\sigma}$ is unbounded, since $\Delta_{G}$ is an unbounded function.

Now let us construct the operator field. We first give the $A(\sigma)$ pointwise and postpone the questions of measurability and square-integrability. Given $\sigma \in \hat{G}$, $A(\sigma)$ lives on $\mathcal{H}_{\sigma}=\mathrm{L}^{2}\left(\Gamma, d \mu_{\Gamma} ; \mathcal{H}_{\tau(\sigma)}\right)$. Pick $c>1$ in such a way that $\{\gamma \in \Gamma$ : $\left.1 \leq \Delta_{G}^{-1 / 2}(\gamma)<c\right\}$ has positive Haar measure, and define, for $n \in \mathbb{N}, S_{n}:=\{\gamma \in$ $\left.\Gamma: c^{n} \leq \Delta_{G}^{-1 / 2}(\gamma)<c^{n+1}\right\}$. Let $\left(u_{n}^{\sigma}\right)_{n \in \mathbb{N}} \subset \mathrm{L}^{2}\left(\Gamma, d \mu_{\Gamma} ; \mathcal{H}_{\tau(\sigma)}\right)$ be an orthonormal basis. Moreover let $\left(v_{n}^{\sigma}\right)_{n \in \mathbb{N}} \subset \mathrm{L}^{2}\left(\Gamma, d \mu_{\Gamma} ; \mathcal{H}_{\tau(\sigma)}\right)$ be a sequence of unit vectors with $\operatorname{supp}\left(v_{n}^{\sigma}\right) \subset S_{n}$. Define the linear operator $A(\sigma)$ by

$$
A(\sigma)\left(u_{n}^{\sigma}\right):=\left\|\Delta_{G}^{-1 / 2} v_{n}^{\sigma}\right\|^{-1} v_{n}^{\sigma} .
$$

Then, since $\left\|\Delta_{G}^{-1 / 2} v_{n}^{\sigma}\right\| \geq c^{n}, A(\sigma)$ is a Hilbert-Schmidt operator. In addition, $C_{\sigma} A(\sigma)$ is an isometry, since $A(\sigma)$ maps $\mathcal{H}_{\sigma}$ into $\operatorname{dom}\left(C_{\sigma}\right)$, and $C_{\sigma} A(\sigma)$ maps the orthonormal basis $\left(u_{n}^{\sigma}\right)_{n \in \mathbb{N}}$ to the orthonormal system $\left(\left\|\Delta_{G}^{-1 / 2} v_{n}^{\sigma}\right\|^{-1} \Delta_{G}^{-1 / 2} v_{n}^{\sigma}\right)_{n \in \mathbb{N}}$. Finally, $A(\sigma)^{*} C_{\sigma}$ has a continuous extension: To see this, let $f \in \operatorname{dom}\left(C_{\sigma}\right) . \quad f$ decomposes in the orthogonal sum $f=g+\sum_{n \in \mathbb{N}} f_{n}$, where $f_{n}$ has support in $S_{n}$, and $g$ vanishes on the $S_{n}$. By assumption, $\Delta_{G}^{-1 / 2} f \in \mathrm{L}^{2}\left(\Gamma, d \mu_{\Gamma} ; \mathcal{H}_{\tau(\sigma)}\right)$. The operator $A(\sigma)^{*}$ is given by

$$
\begin{aligned}
A(\sigma)^{*}\left(v_{n}^{\sigma}\right) & =\left\|\Delta_{G}^{-1 / 2} v_{n}^{\sigma}\right\|^{-1} u_{n}^{\sigma}, \\
A(\sigma)^{*}(w) & =0, \text { whenever } w \perp\left\{v_{n}^{\sigma}: n \in \mathbb{N}\right\} .
\end{aligned}
$$

Hence

$$
A(\sigma)^{*} C_{\sigma} f=\sum_{n \in \mathbb{N}}\left\langle\Delta_{G}^{-1 / 2} f_{n}, v_{n}^{\sigma}\right\rangle\left\|\Delta_{G}^{-1 / 2} v_{n}^{\sigma}\right\|^{-1} u_{n}^{\sigma}
$$


Since $\Delta_{G}^{-1 / 2} \leq c^{n+1}$ on the support of $f_{n}$, we have $\left\|\Delta_{G}^{-1 / 2} f_{n}\right\| \leq c^{n+1}\left\|f_{n}\right\|$. On the other hand, $\left\|\Delta_{G}^{-1 / 2} v_{n}^{\sigma}\right\|^{-1} \leq c^{-n}$, hence $\left\|A(\sigma)^{*} C_{\sigma} f\right\| \leq c\|f\|$, which means that $\left[A(\sigma)^{*} C_{\sigma}\right]$ exists. Thus $A(\sigma)$ is as desired.

Let us next address the measurability requirement: With respect to direct integrals, we use the terminology of 9]. Clearly it is sufficient to show that the orthonormal basis $\left(u_{n}^{\sigma}\right)_{n \in \mathbb{N}}$ and the images $\left(\left\|\Delta_{G}^{-1 / 2} v_{n}^{\sigma}\right\|^{-1} v_{n}^{\sigma}\right)_{n \in \mathbb{N}}$ can be chosen measurably, that is, for each $n \in \mathbb{N},\left(u_{n}^{\sigma}\right)_{\sigma \in V}$ and $\left(\left\|\Delta_{G}^{-1 / 2} v_{n}^{\sigma}\right\|^{-1} v_{n}^{\sigma}\right)_{\sigma \in V}$ are measurable vector fields.

First of all, since $\tau(V)$ is standard, there exists a measurable realization of the family $\left(\tau(\sigma), \mathcal{H}_{\tau(\sigma)}\right)_{\sigma \in V}$ [18, Theorem 10.2]. This means that there exist vector fields $\left(\left(e_{k}^{\sigma}\right)_{\sigma \in V}\right)_{k \in \mathbb{N}}$, such that for almost all $\sigma \in V,\left(e_{k}^{\sigma}\right)_{k \in \mathbb{N}}$ is total in $\mathcal{H}_{\tau(\sigma)}$, and the mappings $\sigma \mapsto\left\langle e_{k}^{\sigma}, e_{m}^{\sigma}\right\rangle$ as well as $\sigma \mapsto\left\langle e_{k}^{\sigma}, \tau(\sigma)\left(g_{0}\right) e_{m}^{\sigma}\right\rangle$ are measurable, for all $n, m \in \mathbb{N}$ and all $g_{0} \in G_{0}$. The measurability of any vector field is equivalent to the measurability of its scalar products with the $e_{k}^{\sigma}$. Without loss of generality, we may assume in addition that, for $\nu_{G}$-almost every $\sigma$, the first $\operatorname{dim}\left(\mathcal{H}_{\tau(\sigma)}\right)$ vectors are an orthonormal basis of $\mathcal{H}_{\tau(\sigma)}[9.7 .29,7.30]$, and $e_{k}^{\sigma}=0$ for $k>\operatorname{dim}\left(\mathcal{H}_{\tau(\sigma)}\right)$. Let $\left(a_{n}\right)_{n \in \mathbb{N}}$ be any orthonormal basis of $\mathrm{L}^{2}(\Gamma)$. Then $\left(a_{n} e_{k}^{\sigma}\right)_{n, k}$ is an orthonormal basis of $\mathcal{H}_{\sigma}=\mathrm{L}^{2}\left(\Gamma, d \mu_{\Gamma} ; \mathcal{H}_{\tau(\sigma)}\right)$, except for the zero vectors belonging to the indices $k>\operatorname{dim}\left(\mathcal{H}_{\tau(\sigma)}\right)$. This family makes $\left(\mathcal{H}_{\sigma}\right)_{\sigma \in V}$ a measurable field of Hilbert spaces, and we are going to ensure measurability of the operator fields with respect to this structure.

In order to construct the measurable family $\left(u_{n}^{\sigma}\right)_{n, \sigma}$ of orthonormal bases, we proceed as follows: First note that the sets $V_{\ell}:=\left\{\sigma: \operatorname{dim}\left(\mathcal{H}_{\tau(\sigma)}\right)=\ell\right\}$, for $\ell \in$ $\mathbb{N} \cup\{\infty\}$, are Borel sets [18, Theorem 8.7]. On each $V_{\ell}$, pick a fixed bijection $s_{\ell}: \mathbb{N} \times\{1, \ldots, \ell\} \rightarrow \mathbb{N}$ (where $\left.\{1, \ldots, \infty\}:=\mathbb{N}\right)$. Then letting $u_{s_{\ell}(n, k)}^{\sigma}:=a_{n} e_{k}^{\sigma}$, for $\sigma \in V_{\ell}$, removes the zero vectors. Moreover, on each $V_{\ell}$, the measurability is easily checked, and this is sufficient.

For the construction of the $v_{n}$ we pick any family $\left(b_{n}\right)_{n \in \mathbb{N}} \subset \mathrm{L}^{2}(\Gamma)$ of unit vectors, such that $b_{n}$ is supported in $S_{n}$. Moreover, let $\left(\xi^{\sigma}\right)_{\sigma \in V}$ be a measurable field of unit vectors $\xi^{\sigma} \in \mathcal{H}_{\tau(\sigma)}$, and define $v_{n}^{\sigma}=b_{n} \xi^{\sigma}$. Then

$$
\sigma \mapsto\left\langle\left\|\Delta_{G}^{-1 / 2} a_{n}\right\|^{-1} v_{n}^{\sigma}, a_{n} e_{k}^{\sigma}\right\rangle=\left\|\Delta_{G}^{-1 / 2} a_{n}\right\|^{-1}\left\langle b_{n}, a_{n}\right\rangle\left\langle\xi^{\sigma}, e_{k}^{\sigma}\right\rangle
$$

is measurable by the choice of the $\xi^{\sigma}$. Thus we can construct the operator field in a measurable way.

Finally, let us provide for square-integrability. For this purpose we observe that we may assume the constant $c$ picked above to be $\geq 2$, and then $\|A(\sigma)\|_{2}^{2}<2$. Moreover, if we shift the construction in the sense that $u_{n}^{\sigma} \mapsto\left\|\Delta_{G}^{-1 / 2} v_{n+k}^{\sigma}\right\|^{-1} v_{n+k}^{\sigma}$, for $k>0$, we obtain $\|A(\sigma)\|_{2}^{2}<2^{1-k}$, while preserving all the other properties of $A(\sigma)$. With this in mind, we can easily modify the construction to obtain an element of $\mathcal{B}_{2}^{\oplus}$ : Since $G$ is separable, $\nu_{G}$ is $\sigma$-finite, i.e., $\hat{G}=\bigcup_{n \in \mathbb{N}} \Sigma_{n}$ with the $\Sigma_{n}$ pairwise disjoint and $\nu_{G}\left(\Sigma_{n}\right)<\infty$. Shifting on $\Sigma_{n}$ by $k_{n} \in N$ with $\nu_{G}\left(\Sigma_{n}\right) 2^{-k_{n}}<$ $2^{-n}$ ensures square-integrability without destroying measurability. (The latter is obvious on $\Sigma_{n}$.) Hence we are done, noting that the shifting argument also yields the following fact which sharpens the contrast to the unimodular case, where for a given representation the length of admissible vectors is fixed.

Corollary 2.1. There exist admissible vectors with arbitrarily small or big norm. 


\section{CONCluding REMARKS}

The main purpose of this paper was to establish a link between generalized wavelet transforms and Plancherel theory, and to demonstrate the power of the approach via the existence theorem for admissible vectors. It is quite obvious that for a concrete situation the abstract approach does not automatically give access to the construction of admissible vectors. It can be difficult to establish the fact that a given representation is contained in the regular representation, and even when that is known, obtaining explicit knowledge of the decomposition of the representation under the Plancherel transform might turn out to be another serious obstacle. Nevertheless, the approach yields a new perspective on the construction of wavelet transforms, and there are concrete cases where the link to Plancherel theory can be established. A family of examples are the semidirect products $G=\mathbb{R}^{n} \rtimes H$, and their quasi-regular representations already mentioned in the introduction, and studied, with increasing generality, in the papers [19, 13, 15, 4, 10, 16]. In these cases both the decomposition of the quasi-regular representation and the Plancherel theory of $G$ can be computed explicitly, and it is instructive to view the various admissibility conditions derived for those groups in the light of the abstract approach; see [11] for a detailed exposition.

\section{ACKNOWLEDGEMENTS}

The author thanks the Laboratoire d'Analyse, Topologie et Probabilités in Marseille, and the Department of Mathematics and Statistics of Concordia University, Montreal, for their hospitality. He has profited from stimulating discussions with S.T. Ali. The support by the Deutsche Forschungsgemeinschaft (DFG) under the contract $\mathrm{Fu} \mathrm{402/1}$ is also acknowledged.

\section{REFERENCES}

[1] S. T. Ali, J-P. Antoine and J-P. Gazeau, Coherent States, Wavelets and Their Generalizations, Springer-Verlag, New York, 2000. CMP 2000:07

[2] S.T. Ali, H. Führ and A. Krasowska, Plancherel inversion as unified approach to wavelet transforms and Wigner functions, submitted.

[3] D. Arnal and J. Ludwig, Q.U.P. and Paley-Wiener property of unimodular, especially nilpotent, Lie groups, Proc. Amer. Math. Soc. 125 (1997), 1071-1080. MR 97f:43004

[4] D. Bernier and K. Taylor, Wavelets from square-integrable representations, SIAM J. Math. Anal. 27 (1996), 594-608. MR 97h:22004

[5] G. Bohnke, Treillis d'ondelettes aux groupes de Lorentz, Annales de l'Institut Henri Poincaré 54 (1991), 245-259. MR 93c:22020

[6] A.L. Carey, "Group representations in reproducing kernel Hilberts spaces," Reports in Math. Phys. 14 (1978), 247-259. MR 81f:22006

[7] J. Dixmier, $C^{*}$-Algebras, North Holland, Amsterdam, 1977. MR 56:16388

[8] M. Duflo and C.C. Moore, On the regular representation of a nonunimodular locally compact group, J. Funct. Anal. 21 (1976), 209-243. MR 52:14145

[9] G.B. Folland, A Course in Abstract Harmonic Analysis, CRC Press, Boca Raton, 1995. MR 98c:43001

[10] H. Führ, Wavelet frames and admissibility in higher dimensions, J. Math. Phys. 37 (1996), 6353-6366. MR 97h:42014

[11] H. Führ and M. Mayer: Continuous wavelet transforms from semidirect products: Cyclic representations and Plancherel measure. J. Fourier Anal. Appl., to appear.

[12] A. Grossmann, J. Morlet and T. Paul, Transforms associated to square integrable group representations I: General Results, J. Math. Phys. 26 (1985), 2473-2479. MR 86k:22013

[13] C.J. Isham and J.R. Klauder, Coherent states for $n$-dimensional Euclidean groups $E(n)$ and their application, J. Math. Phys. 32 (1991), 607-620. MR 92g:81074 
[14] Q. Jiang, Wavelet transform and orthogonal decomposition of $\mathrm{L}^{2}$ space on the Cartan domain $B D I(q=2)$, Trans. Am. Math. Soc. 349 (1997), 2049-2068. MR 97h:22005

[15] J.R. Klauder and R.F. Streater, A wavelet transform for the Poincaré group, J. Math. Phys. 32 (1991), 1609-1611. MR 92g:81075

[16] R.S. Laugesen, N. Weaver, G. Weiss and E.N. Wilson, Continuous wavelets associated with a general class of admissible groups and their characterization. J. Geom. Anal., to appear.

[17] R.L. Lipsman: Non-abelian Fourier analysis. Bull. Sci. Math. 98 (1974), 209-233. MR 54:13467

[18] G. Mackey, Borel structure in groups and their duals, Trans. Amer. Math. Soc. 85 (1957), 134-165. MR 19:752b

[19] R. Murenzi, Ondelettes multidimensionelles et application à l'analyse d'images, Thèse, Université Catholique de Louvain, Louvain-La-Neuve, 1990.

[20] N. Tatsuuma, Plancherel formula for non-unimodular locally compact groups, J. Math. Kyoto Univ. 12 (1972), 179-261. MR 45:8777

Zentrum Mathematik, TU München, D-80290 München, Germany

Current address: Institut für Biomathematik und Biometrie, GSF-Forschungszentrum für Umwelt und Gesundheit, Ingolstaedter Landstrasse 1, D-85764 Neuherberg, Germany

E-mail address: fuehr@gsf.de 